\title{
Geophysical Research Letters
}

\author{
RESEARCH LETTER \\ 10.1029/2020GL089747 \\ Key Points: \\ - Clearly, SBS dynamics and spectral \\ features are characterized near \\ third electron gyroharmonics at \\ EISCAT \\ - Qualitative comparison of electron \\ temperature inversion based on SBS \\ agrees with ISR measurement \\ - The asymmetries of SBS and \\ DM are closely correlated with \\ enhanced ion lines and electron \\ temperature near $3 f_{\text {ce }}$
}

Correspondence to:

H. Y. Fu,

haiyang_fu@fudan.edu.cn

Citation:

Fu, H. Y., Jiang, M. L., Wang, K. N., Wu, J., Li, Q. L., Rietveld, M. T., et al. (2020). Electron temperature inversion by stimulated Brillouin scattering during electron gyroharmonic heating at EISCAT. Geophysical Research Letters, 47, e2020GL089747. https://doi.org/10.1029/2020GL089747

Received 15 JUL 2020

Accepted 22 AUG 2020

Accepted article online 25 AUG 2020

(C)2020. American Geophysical Union. All Rights Reserved.

\section{Electron Temperature Inversion by Stimulated Brillouin Scattering During Electron Gyroharmonic Heating at EISCAT}

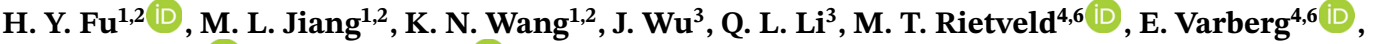 \\ I. Häggström ${ }^{5}$, and Y.-Q. Jin ${ }^{1,2}$ \\ ${ }^{1}$ Key Laboratory for Information Science of Electromagnetic Waves (MoE), Fudan University, Shanghai, China, ${ }^{2}$ School \\ of Information Science and Technology, Fudan University, Shanghai, China, ${ }^{3}$ China Research Institute of Radiowave \\ Propagation, Qingdao, China, ${ }^{4}$ EISCAT Scientific Association, Ramfjordmoen, Norway, ${ }^{5}$ EISCAT Scientific Association, \\ Kiruna, Sweden, ${ }^{6}$ UiT The Arctic University of Norway, Tromsø, Norway
}

\section{Introduction}

The interaction of high-power high-frequency (HF) waves with the ionosphere can excite plasma turbulence and stimulated electromagnetic emissions (SEEs) due to the parametric decay instability process. The complexity of SEE arises from the wave-wave and wave-particle interaction mechanism in magnetized ionospheric plasmas (Leyser, 2001), particularly near electron gyroharmonic $n f_{\text {ce }}$ (Stubbe et al., 1994). The SEE spectra are capable of sensing the interaction mechanism and parameters in the ionospheric resonance regime (Stubbe \& Kopka, 1990). During the past two decades, substantial progress has been achieved for narrow-band SEE with frequency offset of $\sim 2 \mathrm{kHz}$ at $\operatorname{HAARP}\left(62.4^{\circ} \mathrm{N}, 145.2^{\circ} \mathrm{W}\right)$, EISCAT $\left(69.6^{\circ} \mathrm{N}, 19.2^{\circ} \mathrm{W}\right)$, and Arecibo $\left(18.3^{\circ} \mathrm{N}, 66.8^{\circ} \mathrm{W}\right)$. One of the important SEE features is the observation of stimulated Brillouin scattering (SBS) for diagnosing tools. The narrow-band SEE observations have complemented conventional SEE spectrum.

The SBS, arising from phenomenon in optics, has been well studied in unmagnetized plasma (Kruer, 1988). The wave matching conditions for SBS in magnetized ionospheric plasmas (MSBS) (Bernhardt et al., 2010; Norin et al., 2009) are $\omega_{0}=\omega_{\mathrm{S}}+\omega_{\mathrm{L}}$ and $\mathbf{k}_{0}=\mathbf{k}_{\mathrm{s}}+\mathbf{k}_{\mathrm{L}}$, where $\omega$ is the wave frequency, $\mathbf{k}$ is the wave propagation vector, and the subscripts $0, \mathrm{~s}$, and $\mathrm{L}$ denote the pump, scattered, and low-frequency waves, respectively.

The first experimental detection of the ion acoustic (IA) process during ionospheric modification experiment was reported by Norin et al. (2009) using the HAARP transmitter. Subsequent experiments by Bernhardt et al. (2009) provides measurement of the electron temperature by IA in the heated region and predicted electrostatic ion cyclotron (EIC) emission due to MSBS. The EIC emission was successfully measured at the HAARP facility with oblique waves by Bernhardt et al. (2010). At EISCAT, Fu et al. (2015) observed the IA SBS emission by ordinary (O) mode near $3 f_{\text {ce }}$. Afterwards, Blagoveshchenskaya et al. (2015) also observed IA and EIC emissions due to MSBS by extraordinary (X) mode at EISCAT. Mahmoudian, Senior, et al. (2019) observed IA and EIC emissions due to MSBS near $4 f_{\text {ce }}$. At Arecibo, SBS emissions including IA and EIC have also been observed by Djuth et al. (2018) and Mahmoudian, Nossa, et al. (2019). Meanwhile, the correlation 
between SEE with electron temperature, plasma irregularities, HF-induced plasma, and ion lines has also been investigated at EISCAT (Honary et al., 1995) and HAARP (Fu et al., 2018; Mahmoudian et al., 2016).

Ionospheric parameter inversion by SEE has been pursued as complementary diagnostic tool for the incoherent scatter radar (ISR). The electron temperature inversion based on SBS has been becoming the most promising candidate. However, quantitative characteristics of SBS and its inversion for electron temperature in comparison with the ISR have not been thoroughly investigated near $3 f_{\text {ce }}$. This letter reports a pump wave frequency sweeping experiment near $3 f_{\text {ce }}$ at EISCAT to investigate the dynamics of SBS spectra with simultaneous measurement of electron temperature by the ISR. This paper is organized as follows. In section 2, experimental procedure and diagnostics are described at EISCAT. Section 3 describes experimental observations and analysis of SEE and electron temperature inversion by SBS in comparison to the ISR. Section 4 summarizes conclusions.

\section{Experiment Setup}

The experiment at EISCAT was conducted on 25 November 2019 with the HF transmitter operating at $\mathrm{O}$-mode polarization with full power. The beam was transmitted in a quasi-continuous wave mode along the magnetic field. For one time cycle within $18 \mathrm{~min}$, the HF pump wave frequency is turned on at $3.9 \mathrm{MHz}$ for the first $2 \mathrm{~min}$ and stepped by $3.125 \mathrm{kHz}$ every $10 \mathrm{~s}$ for the $16 \mathrm{~min}$ remaining. The pump wave frequency steps through the third electron gyroharmonic $3 f_{\text {ce }}$. The effective radiated power was approximately 138 MW. Two cycles I and II were conducted successfully before the maximum peak density foF2 of background ionosphere dropped substantially below $3 f_{\text {ce }}$.

The SEE receiver was installed in Kroken, a suburb of Tromsø, about $13 \mathrm{~km}$ north-northwest (NNW) of the heater facility in Ramfjordmoen, separated by a mountain range. The antenna was a Wellbrook active loop antenna. A dechirping algorithm is adopted in the time domain to remove the pump frequency. The sampled data are processed with the short-time Fourier transform (STFT) to yield low-frequency spectra of the backscatter wave sidebands around the pump frequency.

The EISCAT $931 \mathrm{MHz}$ ultrahigh frequency (UHF) radar was operated in the Beata mode, which enables measurement from 70 to $680 \mathrm{~km}$ with a minimum of 5-s time resolution and $3.5-\mathrm{km}$ range resolution. The UHF radar data were integrated for $10 \mathrm{~s}$ with approximately $14-\mathrm{km}$ resolution near the reflection region. The UHF ISR can provide ionospheric measurement, including electron temperature $T_{\mathrm{e}}$, electron density $n_{\mathrm{e}}$, electron and ion temperature ratio $T_{\mathrm{e}} / T_{\mathrm{i}}$, and plasma flow velocity $V_{\mathrm{d}}$. A dynasonde colocated with EISCAT with a sounding every 2 min provides the bottomside electron density of the ionosphere.

\section{Experimental Results and Analysis}

Figure 1 depicts the spectrogram of scattered electromagnetic waves from the HF pump at EISCAT during universal time (UT) from 10:45:10 to 11:03:10 for Cycle I and from 11:15:00 to 11:33:00 for Cycle II on 25 November 2019. The three pairs of panels in Figure 1 show wideband spectrograms within $20 \mathrm{kHz}$ (top), narrowband SBS within $100 \mathrm{kHz}$ (middle), and one-dimensional averaged power and frequency offset for SBS emissions, respectively. The top panels of Figure 1 show wideband spectrograms within $20 \mathrm{kHz}$ including prominent downshifted maximum (DM) and downshifted peak (DP). The evolution of wideband SEE within $18 \mathrm{~min}$ shows that DM features with frequency offset -11.5 to $-7.4 \mathrm{kHz}$ start to develop and become suppressed at 10:55:00 and 11:25:00. It is noted that the DP frequency appears at $-2 \mathrm{kHz}$ from 10:55 and gradually decreases to $\sim-0.85 \mathrm{kHz}$ at 10:58 for Cycle I, which shows similar trends to that in Cycle II. For pumping above $3 f_{\text {ce }}$, both DM and second DM start to become strong.

In the middle panel of Figure 1, the narrowband spectra shows the SBS emissions at approximately $-30 \mathrm{~Hz}$ and upshifted emission at $\sim 30 \mathrm{~Hz}$. These emissions are SBS-2 from the upper hybrid region. The SBS-1 may be also observed for Cycle I with frequency offset below $15 \mathrm{~Hz}$. For simplicity, we refer to SBS as SBS-2 unless stated. The power line frequency is $\pm 50 \mathrm{~Hz}$. For Cycle I, the downshifted SBS appears for 4.0-4.041 $\mathrm{MHz}$ below $3 f_{\text {ce }}$ at $t=431-570 \mathrm{~s}$. Both downshifted and upshifted SBS are observed for 4.144-4.2 MHz at $t>891 \mathrm{~s}$ above $3 f_{\mathrm{ce}}$. It is observed that SBS emissions are suppressed for pumping near $3 f_{\mathrm{ce}}$. For Cycle II, spectral features below $\sim 20 \mathrm{~Hz}$ have also been observed and strengthened near $3 f_{\text {ce }}$, that may arise from an IA wave as SBS-1 from the reflection altitude (Fu et al., 2015). The power and spectral width of downshifted SBS emissions increase for pumping above $3 f_{\text {ce }}$. Importantly, it is newly observed that SBS evolves similarly 

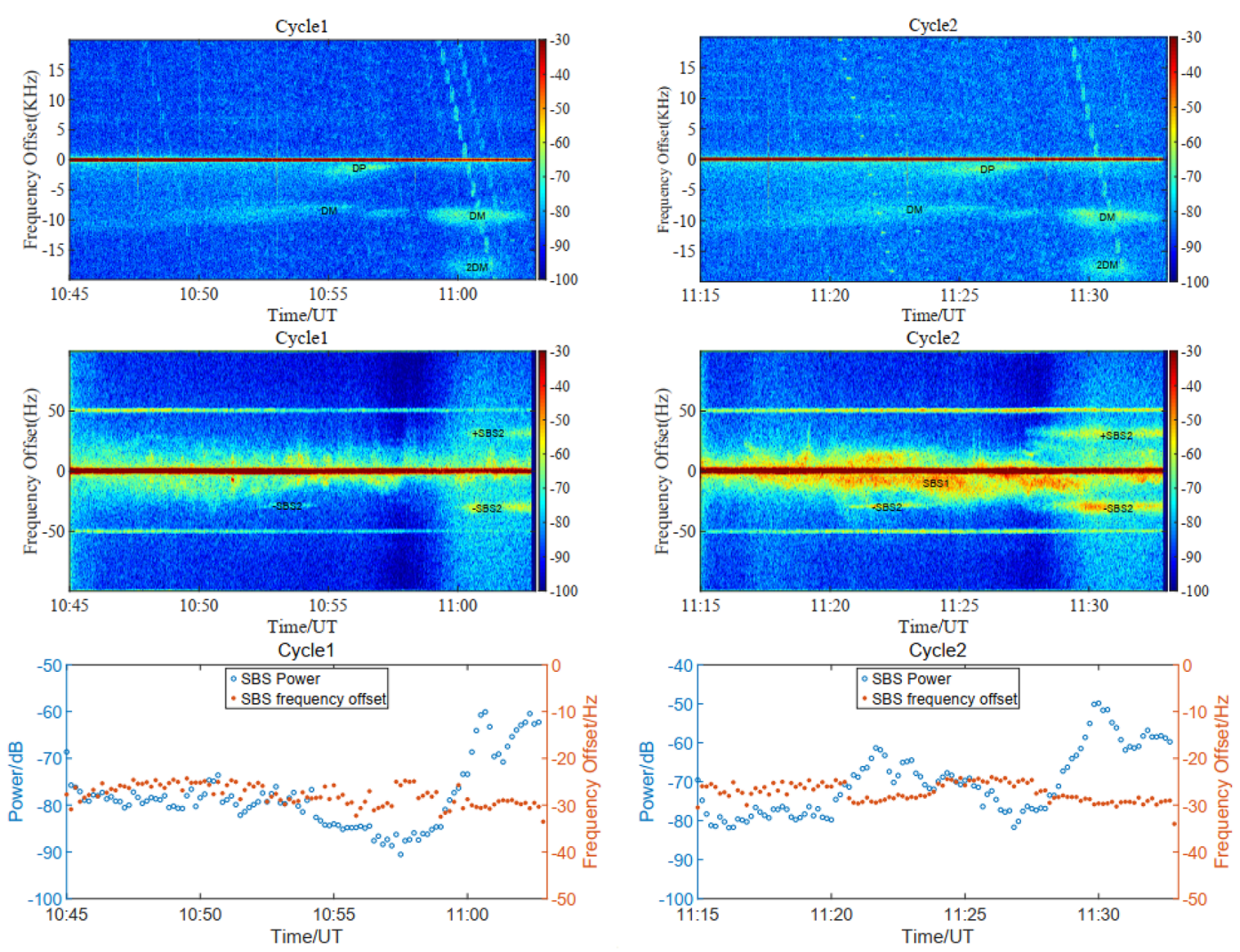

Figure 1. Experimental frequency spectra of scattered waves for HF pump wave frequency sweeping near the third electron gyroharmonic $3 f_{\text {ce }}$ at EISCAT on 25 November 2019, including (1) wideband SEE within $20 \mathrm{kHz}$ showing DM and DP (top), (2) narrowband SEE within $100 \mathrm{~Hz}$ (middle) showing SBS, and (3) one-dimensional SBS averaged frequency offset and power (bottom).

to DM due to different parametric decay processes, both of which are considered to arise from the upper hybrid resonance level.

The averaged power and frequency offset of SBS emissions near $3 f_{\text {ce }}$ are depicted in the bottom panel of Figure 1. Here, the averaged frequency shift of SBS is calculated based on $f=\sum_{i=1}^{N=5} P_{\mathrm{i}} f_{\mathrm{i}} /\left(P_{1}+P_{2}+P_{3}+\right.$ $P_{4}+P_{5}$ ), where five frequency points are $F_{1}, F_{2}, F_{3}, F_{4}$, and $F_{5}$ with maximum power $P_{1}, P_{2}, P_{3}, P_{4}$, and $P_{5}$ within the frequency band of -40 to $-23 \mathrm{~Hz}$. It is clearly observed that SBS power has 20-dB enhancement for pumping above electron gyroharmonic. For Cycle II, it is clearly observed that the SBS frequency shift decreases from UT time 11:21 to 11:24 below $3 f_{\mathrm{ce}}$ and increases from 11:27 to 11:30 above $3 f_{\mathrm{ce}}$. The asymmetries of SBS power and spectral width are observed for pumping above $3 f_{\mathrm{ce}}$ for Cycles I and II.

Figure 2 shows measurement of ionospheric plasma parameters by the ISR for HF pump wave frequency sweeping near $3 f_{\text {ce }}$ at EISCAT on 25 November 2019 for each panel: (1) electron density $n_{\mathrm{e}}$; (2) residual; (3) electron temperature $T_{\mathrm{e}}$; and (4) raw electron density. The time resolution of the ISR is $30 \mathrm{~s}$. The first panel of Figure 2 shows electron density, which shows little change except for around 11:03 and 11:33 at the resonance range gates 200 and $214 \mathrm{~km}$.

The second panel of Figure 2 shows the residual error, which is a measure of how good the theoretical spectra fit to the data. The fitting is good for residual value $\sim 1$ and becomes not reliable for value $\sim 10$. Therefore, the electron density and temperature during intervals around 11:03 and 11:33 may not be reliable since the enhanced backscatter from HF-enhanced ion lines is caused by the parametric decay instabilities.

The third panel of Figure 2 shows that electron temperature increases from 2,000 to 4,000 $\mathrm{K}$ at ranges from 200 to above $300 \mathrm{~km}$. The electron temperature enhancement is suppressed near $3 f_{\mathrm{ce}}$ for both cycles. For Cycle II (11:15-11:33), electron temperature shows substantial asymmetry with $T_{\mathrm{e}}$ enhancement from 2,000 to $4,000 \mathrm{~K}$ for $f_{0}<3 f_{\text {ce }}$ and from 2,000 to $6,000 \mathrm{~K}$ for $f_{0}>3 f_{\text {ce }}$, that will be illustrated in Figure 3 . 

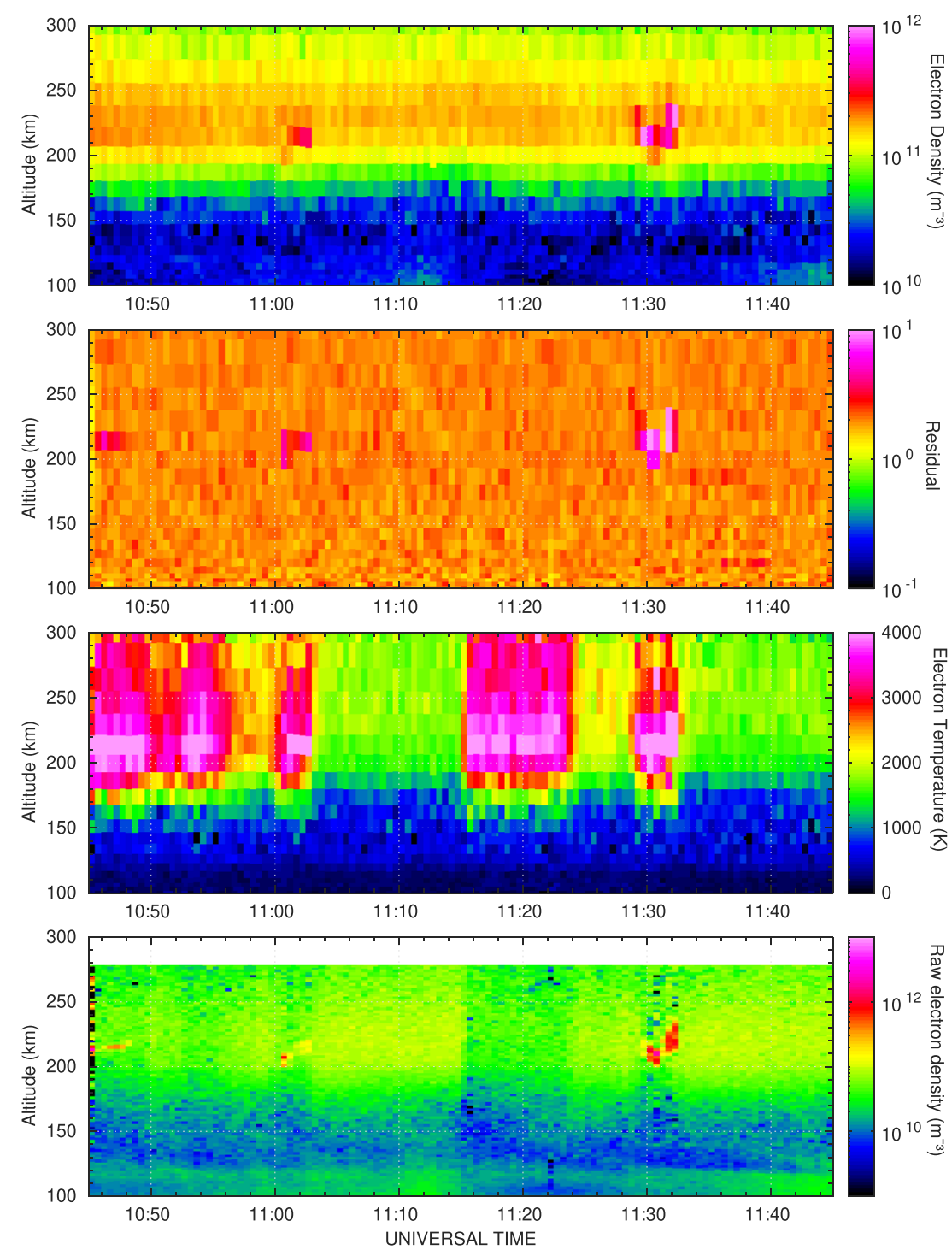

Figure 2. Simultaneous measurement of ionospheric plasma parameters by the EISCAT UHF radar for HF pump wave frequency sweeping near $3 f_{\text {ce }}$ at EISCAT for Cycle I (10:45-11:03) and for Cycle II (11:15-11:33) on 25 November 2019. Each panel shows (1) electron density $n_{\mathrm{e}}$; (2) residual; (3) electron temperature $T_{\mathrm{e}}$; and (4) raw electron density, respectively.

The fourth panel of Figure 2 shows the raw electron density that is just backscattered power multiplied by a constant to convert it to units of electron density. It is observed that for pumping above gyroharmonic during 11:03 UT for Cycle II, the raw electron density shows HF enhanced ion lines (HFILs) at the resonance range gates 200 and $214 \mathrm{~km}$.

During the heating cycles I and II, ion temperature stays almost the same, approximately at $800 \mathrm{~K}$. It is worthwhile noticing that a correlation of an electron density depletion was observed along with strong electron temperature enhancement and ion temperature enhancement for plasma cavity formation during a heating experiment at Arecibo observatory by Levine et al. (2020).

Figure 3 shows the average power of SEE and electron temperature $T_{\mathrm{e}}$ at resonance altitudes 200 and 214 km (upper) and HFILs (lower) from UT time 10:45 to 11:33 on 25 November 2019 near $3 f_{\text {ce }}$. The time resolution of the ISR is $10 \mathrm{~s}$. Using the International Geomagnetic Reference(IGRF) model, the third electron gyroharmonic frequency is approximately estimated to be $3 f_{\text {ce }} \sim 4.1038 \mathrm{MHz}$ at $215 \mathrm{~km}$ and $3 f_{\text {ce }} \sim 4.1275$ $\mathrm{MHz}$ at $200 \mathrm{~km}$. As an indicator of pump frequency near $3 f_{\mathrm{ce}}$, the DP, DM, and SBS reach a minimum at UT time 10:57 and 10:58 for Cycle I and 11:27 and 11:28 for Cycle II as shown in the black dash box. For Cycle 


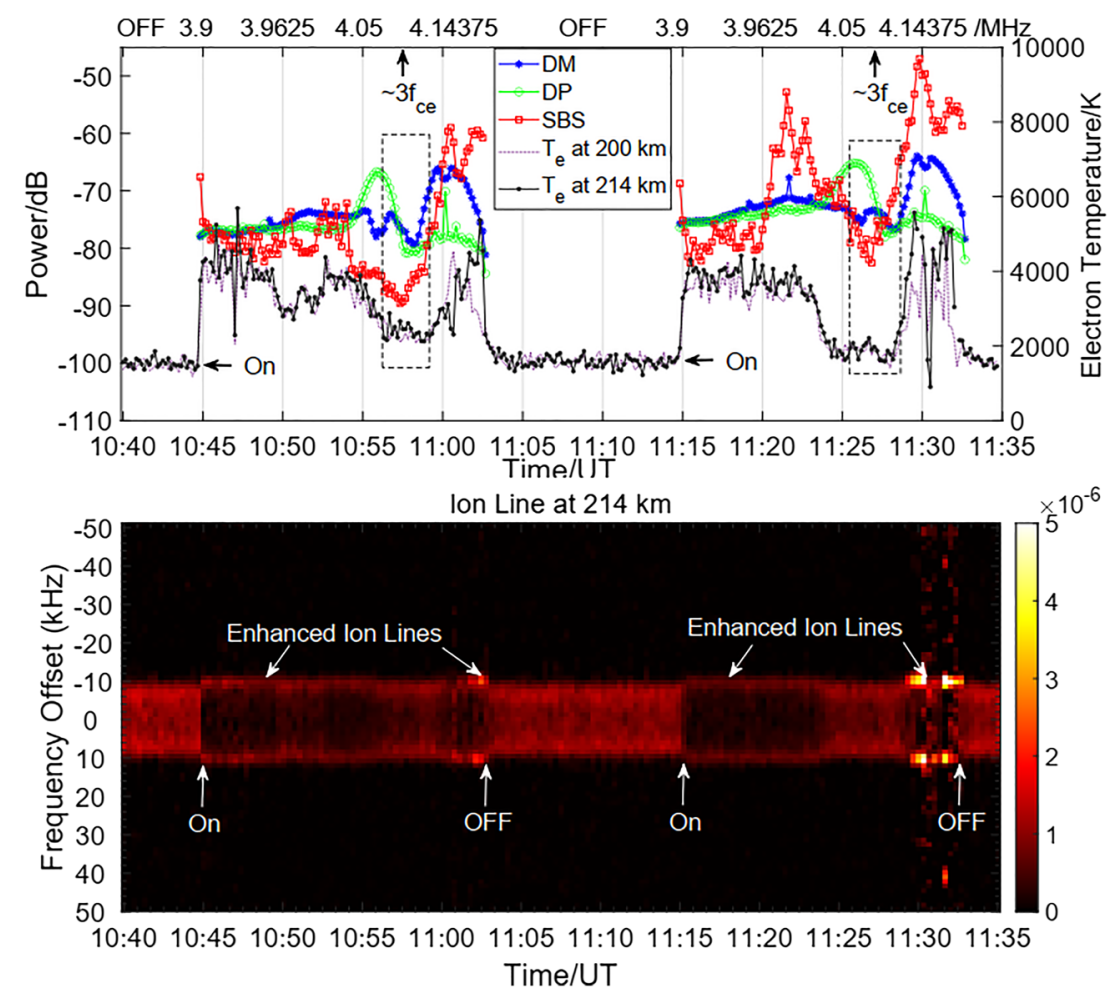

Figure 3. Simultaneous measurement of SBS, DP, DM with electron temperature $T_{\mathrm{e}}$ (upper), and HF-enhanced ion lines (lower) for HF pump wave frequency sweeping near the third electron gyroharmonic $3 f_{\text {ce }}$ at EISCAT on 25 November 2019. The dashed box shows SEE features and $T_{\mathrm{e}}$ near $3 f_{\mathrm{ce}}$ for Cycle I (10:45-11:03) and for Cycle II (11:15-11:33). The enhanced asymmetries of SBS, DM, $T_{\mathrm{e}}$, and enhanced ion lines are observed for $f_{0} \geq 3 f_{\mathrm{ce}}$ for Cycles I and II.

I (II), spectral power reaches minimum for SBS 4.1 MHz (4.0906 MHz), DP 4.1094 MHz (4.1063 MHz), and $\mathrm{DM} \sim 4.1156 \mathrm{MHz}(4.1156 \mathrm{MHz})$.

Another important feature is that SBS power and $T_{\mathrm{e}}$ as well as the DM strength decrease substantially for the pump frequency below $3 f_{\text {ce }}$ and increase above $3 f_{\text {ce }}$. It is newly observed that correlation between SBS and $T_{\mathrm{e}}$ was exhibited. SBS shows similar features as DM with $T_{\mathrm{e}}$ showing opposite behavior to the DP near $3 f_{\mathrm{ce}}$. The SBS power can achieve approximately 20-dB gain compared to the DM. Thus, it is natural to question which mechanism (DM or SBS) plays an important role for electron temperature enhancement.

The lower panel of Figure 3 shows the strength of HFILs at the resonance altitude $214 \mathrm{~km}$ near $3 f_{\text {ce }}$. When the heater is turned on, the ion lines are enhanced. For pumping $f_{0}-3 f_{\text {ce }}$, the ion line enhancement damps out. For $f_{0} \geq 3 f_{\text {ce }}$, strong ion line enhancement is observed particularly at $t=11: 30-11: 33$ during Cycle II. It seems that the ion line spectra become spread for $t=11: 30-11: 33$ during Cycle II. Recently, Yellu et al. (2020) investigated stimulated Brillouin backscattering during ionospheric heating by a kinetic model that shows ion density cavity development and electron temperature enhancement by a factor of $\sim 5$. Based on the correlation between SBS and DM, it leads us to believe that DM may be also utilized for electron temperature retrieval as attempted in Fu et al. (2020).

Figure 4 shows the pump beam transmitted along the magnetic field with pump frequency near $3 f_{\text {ce }}$ on 25 November 2019 in the upper panel. The foF 2 of ionospheric density are retrieved based on measurement by the dynasonde every 2 min and the EISCAT UHF incoherent scatter radar near Troms $ø$ every $10 \mathrm{~s}$. It is noted that when the heater is turned off, the peak frequency foF2 satisfies the overdense condition before 10:45 for Cycle I and 11:15 for Cycle II. After heating is turned on, the foF2 peak measurement by dynasonde and ISR radar seems to drop from 10:45 to 10:55 and 11:15 to 11:25 and starts to increase from UT 11:00 to 11:03 and UT 11:30 to 11:33. Although the inversion accuracy of electron density during heating by the dynasonde and ISR is still questionable, the electron density seems to be suppressed and then increases for pumping above $3 f_{\text {ce. }}$. 

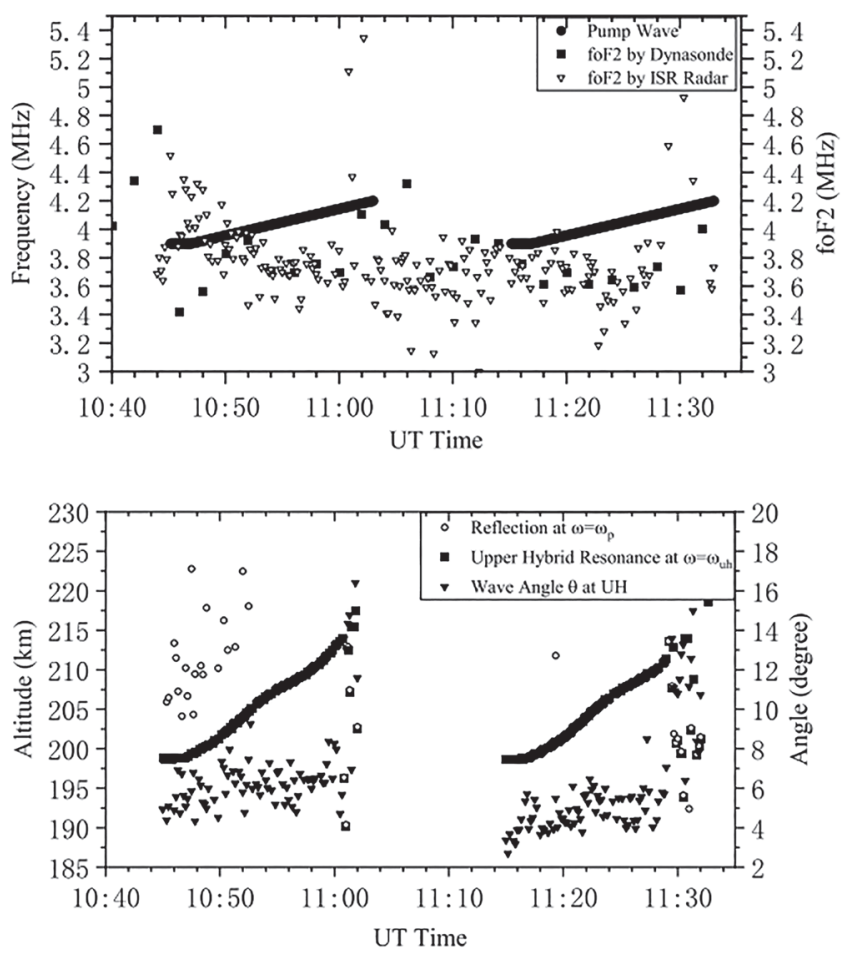

Figure 4. HF Pump wave frequency for varying foF2 (upper) and ray tracing for wave propagation angle $\theta$ (lower) at the upper hybrid resonance height on 25 November 2019. It is noted that the foF2 satisfies the overdense heating condition before the heater turns on at UT 10:45 for Cycle I and at UT 11:15 for Cycle II.
The lower panel of Figure 4 shows the results of ray tracing for magnetic zenith for which the $\mathrm{O}$ mode is reflected at $\omega_{0}=\omega_{\mathrm{p}}$, where $\omega_{\mathrm{p}}$ is the plasma wave frequency. The upper hybrid wave resonance height is calculated based on the upper hybrid wave frequency $\omega_{\mathrm{UH}}=\sqrt{\omega_{0}^{2}-\Omega_{\mathrm{e}}^{2}}$, where $\Omega_{\mathrm{e}}$ is the electron gyrofrequency. The wave propagation angle $\theta$ at the upper hybrid resonance level where $\omega_{0}=\omega_{\mathrm{uh}}$ is calculated for the upgoing magnetic zenith ray. Based on ray tracing in Figure 4, the wave angle $\theta$ is calculated and averaged to be $6^{\circ}$ for UT time 10:45-10:51 for the foF 2 density above pump wave frequency. Here, electron density by the ISR every $10 \mathrm{~s}$ is adopted rather than dynasonde $(2 \mathrm{~min}$ ) for ray tracing as reference. It is noted that for abrupt change in electron density leads to decreased resonance height and increased angles to $16^{\circ}$ at 11:00-11:03 for Cycle I and at 11:30-11:33 for Cycle II. The reflection altitude and the UH resonance height overlaps, which drops from 214 to $190 \mathrm{~km}$ due to electron density steepening based on ISR measurement in Figure 2.

Based on the wave matching condition, the IA speed $c_{\mathrm{IA}}$ at the upper hybrid (UH) matching altitude for the O mode by Bernhardt et al. (2009) is

$$
c_{\mathrm{IA}}=\frac{c \omega_{\mathrm{IA}}}{2 \sqrt{\Omega_{\mathrm{e}} \omega_{0}}} \sqrt{\frac{\Omega_{\mathrm{i}}^{2}-\omega_{0}^{2}}{\Omega_{\mathrm{i}}^{2} \cos ^{2} \theta-\omega_{0}^{2}}} \sqrt{\frac{\omega_{0}+\Omega_{\mathrm{e}} \cos \theta}{\omega_{0} \cos \theta+\Omega_{\mathrm{e}}}}
$$

where $\omega_{\text {IA }}$ is the IA wave frequency and $\Omega_{\mathrm{i}}$ is ion gyrofrequency. Here, the angle $\theta$ is between the wave vector $\mathbf{k}_{\mathrm{IA}}$ with respect to the background magnetic field $\mathbf{B}$ as $\mathbf{B} \cdot \mathbf{k}_{\mathrm{IA}} \cdot=|\mathbf{B}|\left|k_{\mathrm{IA}}\right| \cos \theta$. The IA wave speed $c_{\mathrm{IA}}$ depends on the electron and ion temperature as

$$
c_{\mathrm{IA}}=\sqrt{\frac{\gamma_{\mathrm{e}} T_{\mathrm{e}}+\gamma_{\mathrm{i}} T_{\mathrm{i}}}{m_{\mathrm{i}}}}
$$

where $\gamma_{\mathrm{e}}=1$ and $\gamma_{\mathrm{i}}=3$ for isothermal plasmas.

Figure 5 shows electron temperature $T_{\mathrm{e}}$ and ion temperature $T_{\mathrm{i}}$ inversion based on SBS at the resonance height in comparison with measurement by the ISR radar on 25 November 2019. Based on Equations 1 and 2, electron temperature can be retrieved assuming the ratio of $T_{\mathrm{e}} / T_{\mathrm{i}}$ is known with the wave angle $\theta$ calculated from ray tracing in Figure 4. Here, we compare electron temperature at the UH resonance height $\sim 200$ and $214 \mathrm{~km}$ for $T_{e} / T_{i}=3$ which is commonly used and for $T_{\mathrm{e}} / T_{\mathrm{i}}$ as measured independently by the ISR.
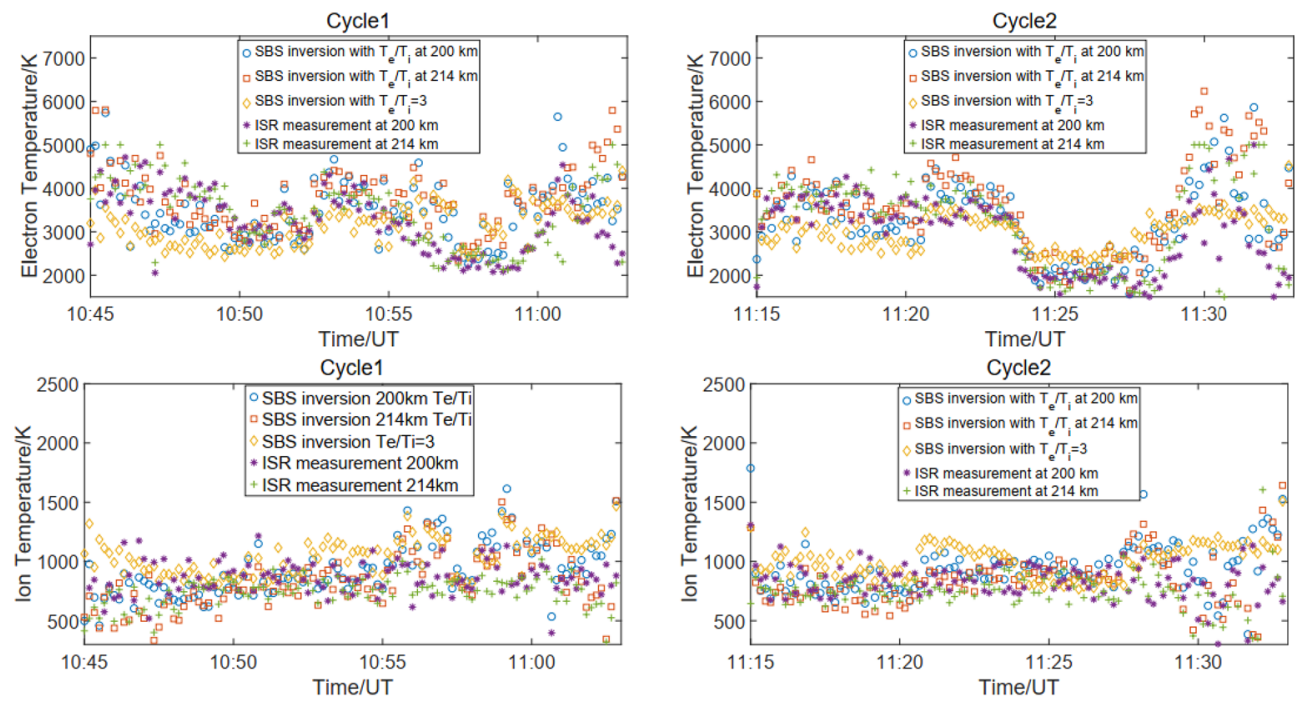

Figure 5. Electron temperature $T_{\mathrm{e}}$ and ion temperature $T_{\mathrm{i}}$ inversion based on stimulated Brillouin scattering (SBS) in comparison with measurement by incoherent scatter radar (ISR) on 25 November 2019. The ratio of $T_{\mathrm{e}} / T_{\mathrm{i}}$ is compared for 3 and value by ISR measurement at resonance heights 200 and $214 \mathrm{~km}$. 
The top panel of Figure 5 shows electron temperature inversion based on SBS for two cycles. Assuming $T_{e} / T_{i}=3$, it can estimate electron temperature for pumping below but cannot capture the asymmetry for $f_{0} \geq 3 f_{\text {ce }}$. However, for $T_{\mathrm{e}} / T_{\mathrm{i}}$ measured from ISR, electron temperature $T_{\mathrm{e}}$ agrees with ISR measurement well at two resonance altitude. Ion temperature inversion based on SBS is also compared with the ISR with good agreement. Based on measurement of $T_{\mathrm{e}} / T_{\mathrm{i}}$ by ISR, we do not observe clear ion temperature change when SBS spectral offset and width intensify.

\section{Conclusions}

This paper has provided experimental observation and parameter inversion of stimulated Brillouin scatter near $3 f_{\text {ce }}$ at EISCAT. It is observed that the evolution of narrowband SBS with $\sim 30 \mathrm{~Hz}$ offset from pump behaves in a similar way to the DM emissions, which provides additional evidence of resonance at the upper hybrid level. Experimental observations show that the SBS power $(\sim 30 \mathrm{~Hz})$ is suppressed and the frequency offset gradually decreases as the pump frequency approaches close to $3 f_{\mathrm{ce}}$. It is concluded that the asymmetries of SBS, DM, electron temperature, and HFILs are observed for $f_{0} \geq 3 f_{\text {ce }}$ at EISCAT.

The inversion of electron temperature and ion temperature based on SBS has been calculated and compared with the ISR measurement. A weighting algorithm is proposed to estimate the SBS spectral offset for electron temperature inversion. The inversion results for varying ratio $T_{\mathrm{e}} / T_{\mathrm{i}}$ show good agreement with the ISR measurement. However, the ISR scatter theory at the resonance regime has been argued inaccurate due to purely Maxwellian distribution. The spectral width enhancement of SBS has not been used-this will be investigated in the future.

\section{Data Availability Statement}

The data used in this paper are available through the EISCAT website (https://www.eiscat.se/scientist/ data/).

\section{References}

\section{Acknowledgments}

The authors would like to acknowledge the EISCAT facility. EISCAT is an international association supported by research organizations in China (CRIRP), Finland (SA), Japan (NIPR and ISEE), Norway (NFR), Sweden (VR), and the United Kingdom (UKRI). The authors would like to acknowledge the staff at the EISCAT facility for technical support and also Tong Xu, Jutao Yang, and Bin $\mathrm{Xu}$ from CRIRP for arranging this campaign. This work is supported by the Shanghai Science and Technology Committee under grant 19511100600 and grant 19ZR1403900.
Bernhardt, P. A., Selcher, C. A., Lehmberg, R. H., Rodriguez, S. P., Thomason, J. F., Groves, K. M., et al. (2010). Stimulated Brillouin scatter in a magnetized ionospheric plasma. Physical Review Letters, 104(165004), 1-4.

Bernhardt, P. A., Selcher, C. A., Lehmberg, R. H., Rodriguez, S., Thomason, J., McCarrick, M. J., \& Frazer, G. J. (2009). Determination of the electron temperature in the modified ionosphere over HAARP using the HF pumped stimulated Brillouin scatter (SBS) emission lines. Annales Geophysicae, 27(12), 4409-4427.

Blagoveshchenskaya, N. F., Borisova, T. D., Yeoman, T. K., Hggstrm, I., \& Kalishin, A. S. (2015). Modification of the high latitude ionosphere $\mathrm{F}$ region by X-mode powerful HF radio waves: Experimental results from multi-instrument diagnostics. Journal of Atmospheric and Solar-Terrestrial Physics, 135, 50-63.

Djuth, F. T., Bernhardt, P. A., \& Zhang, L. (2018). Magnetized stimulated Brillioun scatter excited in the F region and sporadic E at Arecibo Observatory. Triennial Earth-Sun Summit, 262, 20-24.

Fu, H. Y., Jiang, M. L., Wu, J., Li, Q. L., \& Rietveld, M. T. (2020). Electron temperature prediction by downshifted maximum in stimulated electromagnetic emissions via neural network. In 33nd URSI General Assembly and Scientic Symposium, 2021, Rome. https://www. ursi.org/proceedings/procGA20/papers/URSIJMLv4.pdf

Fu, H. Y., Scales, W. A., Bernhardt, P. A., Briczinski, S. J., Kosch, M. J., Senior, A., et al. (2015). Stimulated Brillouin scattering during electron gyro-harmonic heating at EISCAT. Annales Geophysicae, 33, 983-990.

Fu, H. Y., Scales, W. A., Bernhardt, P. A., Jin, Y. Q., \& Briczinski, S. J. (2018). Asymmetry in stimulated emission polarization and irregularity evolution during ionospheric electron gyroharmonic heating. Geophysical Research Letters, 45, 9363-9371. https://doi.org/ 10.1029/2018GL078957

Honary, F., Stocker, A. J., Robinson, T. R., Jones, T. B., \& Stubbe, P. J. (1995). Ionospheric plasma response to HF radio waves operating at frequencies close to the third harmonic of the electron gyrofrequency. Journal of Geophysical Research, 100, 21,489-21,501.

Kruer, W. L. (1988). The physics of laser plasma interactions. Redwood City MA: Addison-Wesley.

Levine, E. V., Bernhardt, P. A., Sulzer, M. P., Sultan, P. J., Henderson, B. S., \& Nossa, E. (2020). Plasma cavity formation during ionospheric heating at Arecibo. Journal of Geophysical Research: Space Physics, 125, e2019JA027715. https://doi.org/10.1029/2019JA027715

Leyser, T. B. (2001). Stimulated electromagnetic emissions by high-frequency electromagnetic pumping of the ionospheric plasma. Space Science Reviews, 98, 223-328.

Mahmoudian, A., Nossa, E., Isham, B., Bernhardt, P. A., Briczinski, S. J., \& Sulzer, M. (2019). NSEE yielding electron temperature measurements at the Arecibo Observatory. Journal of Geophysical Research: Space Physics, 124, 3699-3708. https://doi.org/10.1029/ 2019JA026594

Mahmoudian, A., Scales, W. A., Watkins, B. J., Bernhardt, P. A., Isham, B., Vega-Cancel, O., \& Ruohoniemi, J. M. (2016). Investigation of third gyro-harmonic heating at HAARP using stimulated radio emissions and the MUIR and Kodiak radars. Advances in Space Research, 59, 337-350. https://doi.org/10.1016/j.asr.2016.09.029

Mahmoudian, A., Senior, A., \& Kosch, M. (2019). Investigation of incoherent scatter radar spectra features with stimulated electromagnetic emissions at EISCAT, (Vol. 64, pp. 159-170). https://doi.org/10.1016/j.asr.2019.03.028

Norin, L., Leyser, T. B., Nordblad, E., Thidé, B., \& McCarrick, M. (2009). Unprecedentedly strong and narrow electromagnetic emissions stimulated by high-frequency radio waves in the ionosphere. Physical Review Letters, 102, 65003. https://doi.org/10.1103/PhysRevLett. 102.065003 
Stubbe, P., \& Kopka, H. (1990). Stimulated electromagnetic emission in a magnetized plasma: A new symmetric spectral feature. Physical Review Letters, 65, 183

Stubbe, P., Stocker, A. J., Honary, F., Robinson, T. R., \& Jones, T. B. (1994). Stimulated electromagnetic emissions and anomalous HF wave absorption near electron gyroharmonics. Journal of Geophysical Research, 99(A4), 6233-6246.

Yellu, A. D., Frazier, J. A., \& Scales, W. A. (2020). Simulation studies of strongly turbulent stimulated Brillouin backscattering during ionospheric heating. Radiation Effects and Defects in Solids, 175(1-2), 141-149. 